\title{
Association of first-line antidepressants and incident adverse metabolic effects
}

Frances K. Wen PhD Kimberly Crosby PharmD Barbara H. Miller MD Michael Rommen MD Samuel J. Kirzner MPH Toni Hoberecht MA MLIS AHIP Alyssa Migdalski MLIS

\section{Clinical Inquiries question}

Are first-line antidepressants associated with incident adverse metabolic effects in adults?

\section{Evidence-based answer}

Selective serotonin reuptake inhibitors (SSRIs) and bupropion statistically significantly increase the risk of incident type 2 diabetes mellitus (T2DM) (strength of recommendation [SOR] B: based on a systematic review and meta-analysis, and a prospective cohort study). Selective serotonin reuptake inhibitors and serotonin-norepinephrine reuptake inhibitors (SNRIs) are associated with weight gain, although there is mixed evidence on both its clinical significance and to what degree depression might be a confounding variable (SOR B: based on 3 prospective cohort studies). Evidence suggests there is an association between the development of metabolic syndrome and SSRIs, but it might be dependent upon the choice of diagnostic criteria and SSRI serum concentration or dose (SOR B: based on 2 retrospective cohort studies). No association between SNRIs, bupropion, and incident hypertension persists after adjustment for demographic characteristics, socioeconomic factors, and comorbidities. No association was observed between SSRIs and incident hypertension (SOR B: based on a retrospective cohort study).

\section{Evidence summary}

Diabetes mellitus. A 2017 systematic review and metaanalysis evaluated evidence that supported an association between diabetes and treatment with antidepressant medications (ADMs). ${ }^{1}$ The review and meta-analysis only included studies that assessed the risk of new-onset diabetes (ie, by independent clinical diagnosis, record linkage, and self-report) in adults older than 18 years taking ADMs compared with adults not taking ADMs. Twenty studies-14 cohort studies, 4 nested case-control studies, and 2 randomized controlled trials ( $N=1115125)$ met the criteria for inclusion. Subgroup analyses by ADM type, either SSRI or non-SSRI, found an increased risk of incident diabetes among SSRI users (7 studies; $\mathrm{N}=21511$; relative risk of $1.2 ; 95 \%$ CI 1.1 to 1.4 ).

A 2018 case-noncase disproportionality analysis study, using data from the World Health Organization international database of suspected adverse drug reactions, examined the detection of incident T2DM in those taking ADMs, and compared that to the detection of other adverse drug reactions in ADM users. ${ }^{2}$ Of the 1027405 records that included ADM use, 625127 were selected for analysis. Records involving multiple ADMs, those missing demographic data, and those with age outliers were excluded. The incidence of T2DM was determined for 4 classes of ADMs, SSRIs, tricyclic antidepressants, monoamine oxidase inhibitors, and others, with each class compared to the other 3 using an adjusted reporting odds ratio. A statistically significant incidence of T2DM was observed for SSRIs ( $\mathrm{N}=291020$; adjusted reporting odds ratio of 1.1 ; 95\% CI 1.1 to 1.2 ) and bupropion ( $\mathrm{N}=97557$; reporting odds ratio of 2.0; 95\% CI 1.9 to 2.1 ).

Weight gain. A 2018 population-based cohort study used data from electronic medical records to evaluate the association of long-term ADM exposure on weight gain $(\geq 5 \%)$ throughout the previous year, or long-term ADM exposure on the transition from the overweight classification to the obese classification during 10 years of follow-up. ${ }^{3}$ Participants older than 20 years of age ( $N=294719$; 46\% male) were stratified according to body mass index range at the beginning of the study. Depression status, sex, baseline body mass index, age, diabetes and other concomitant disease states, smoking status, and antiepileptic or antipsychotic medication use were adjusted for in the analysis. There was a significantly increased risk of weight gain associated with SSRIs (N not provided; adjusted rate ratio of 1.21; 95\% CI 1.20 to 1.23) and SNRIs (N not provided; adjusted rate ratio of $1.17 ; 95 \% \mathrm{CI} 1.13$ to 1.21 ).

A 2016 analysis of data from a longitudinal cohort study in the Netherlands examined the relationship between ADM use and a 2-year weight gain in adults 18 to 65 years of age $(\mathrm{N}=2542)$ who met Diagnostic and Statistical Manual of Mental Disorders, 4th edition, criteria for clinical diagnoses of either current or remitted major depressive disorder. ${ }^{4}$ Control participants $(\mathrm{N}=2577)$ had no history of major depressive or anxiety disorders. Data were collected at baseline and every 2 years during a 6 -year period. Compared to the control group, SSRI use $(\mathrm{N}=318)$ was significantly associated with a 2-year weight gain of more than 5\% (odds ratio of 1.3; 95\% CI 1.1 to 1.5). Use of SSRIs was not significantly associated with weight gain after adjusting for depression status.

A 2017 analysis conducted on data collected during an Australian cohort study ( $\mathrm{N}=2334$; mean age of 54 years; $47 \%$ male) examined the association between body weight change and ADM use during a 2- to 6-year follow-up period. ${ }^{5}$ Antidepressant medication use was classified as 
low user (1 to 2 prescriptions/year), high user ( $>2$ prescriptions/year), and nonuser. After adjusting for depression status over time and in comparison to nonusers $(\mathrm{N}=2109)$, an annual weight gain of $0.30 \mathrm{~kg}$ was observed in SSRI low users $(\mathrm{N}=114 ; 95 \% \mathrm{CI} 0.01$ to 0.58$)$ and of $0.48 \mathrm{~kg}$ in high users ( $\mathrm{N}=111$; $95 \% \mathrm{CI} 0.20$ to 0.76$)$; however, these results might not be clinically significant.

Metabolic syndrome. A 2016 analysis of longitudinal data from a French epidemiologic cohort study compared self-reported ADM in adults aged 30 to $65(\mathrm{~N}=5014)$ to the occurrence of metabolic syndrome at 3-year intervals over 9 years. ${ }^{6}$ Antidepressant medication information was provided by participants at each health examination. Metabolic syndrome was defined by the National Cholesterol Education Program Adult Treatment Panel III criteria, and the American Heart Association and the National Heart, Lung, and Blood Institute criteria. Using the National Cholesterol Education Program Adult Treatment Panel III criteria, SSRI use $(\mathrm{N}=253)$ during the 9 years resulted in a 23\% greater annual increase in metabolic syndrome (adjusted odds ratio [aOR] of 1.23; 95\% CI 1.0 to 1.5$)$ compared to nonusers $(\mathrm{N}=15783)$. No significant difference was observed in metabolic syndrome risk between SSRI users and nonusers using the American Heart Association and the National Heart, Lung, and Blood Institute criteria (aOR=1.0; 95\% CI 0.92 to 1.2). Results for both criteria were adjusted for sociodemographic factors, health-related behaviour, medical conditions, and non-SSRI medications.

A 2016 retrospective cross-sectional cohort study of 1301 patients who met the Diagnostic and Statistical Manual of Mental Disorders, 4th edition, criteria for diagnosis of bipolar disorder or schizophrenia, 280 of whom were treated with SSRIs, compared SSRI serum concentrations with changes in cardiovascular risk factors. ${ }^{7}$ Secondary outcomes included occurrence of metabolic syndrome, defined as the presence of 3 or more of the following 5 variables surpassing cutoff levels: waist circumference, high-density lipoprotein cholesterol, triglycerides, glucose, and blood pressure. After adjusting for second-generation antipsychotic medications, the study found that the therapeutic SSRI serum concentration had a statistically significant aOR for metabolic syndrome of 2.1 (95\% CI 1.2 to 3.6). In comparison, no significant association was observed between SSRI dose and metabolic syndrome $(\mathrm{aOR}=1.2 ; 95 \% \mathrm{CI} 0.5$ to 1.4$)$.

Hypertension. A 2018 retrospective cohort study used electronic medical record data of adults with a mean age of 46 years $(\mathrm{N}=6224 ; 63 \%$ female) to examine the association between ADM use and incident hypertension. ${ }^{8}$ Hypertension was defined by the International Classification of Diseases, Ninth Revision, Clinical Modification code 401x. A 3-level ADM variable was created using package insert warnings: ADMs that increase blood pressure (ADM BP+; ie, monoamine oxidase inhibitors, tricyclic antidepressants, SNRIs, and bupropion), ADMs that do not increase blood pressure (ie, all other antidepressants, including SSRIs), and no ADM use. Unadjusted results showed that the ADM BP+ group $(\mathrm{N}=1054)$ was statistically significantly more likely than the no ADM use group ( $\mathrm{N}=4182)$ to develop hypertension (hazard ratio of $1.3 ; 95 \%$ CI 1.1 to 1.6 ). After adjusting for all covariates, there was no longer a statistically significant association between the ADM BP+ group and incident hypertension (hazard ratio of 1.2 ; 95\% CI 0.97 to 1.49). No association was found between the ADMs that do not increase blood pressure group $(\mathrm{N}=1008)$ and incident hypertension for unadjusted or adjusted results.

Dr Wen is Professor, Director of Research, and Behavioral Health Director in the Department of Family and Community Medicine at the University of OklahomaTulsa. Dr Crosby is Associate Professor in the Department of Family and Community Medicine at the University of Oklahoma-Tulsa. Dr Miller is a family physician in Joplin, Mo. Dr Rommen is a family physician in Monroe, La. Mr Kirzner is Double Up Program Manager at Hunger Free Oklahoma in Tulsa. Ms Hoberecht is Technical Services Librarian and Ms Migdalski is Reference and Instruction Librarian in the Schusterman Library at the University of Oklahoma-Tulsa.

Competing interests

None declared

Correspondence

Dr Frances K. Wen; e-mail frances-wen@ouhsc.edu

References

1. Salvi V, Grua I, Cerveri G, Mencacci C, Barone-Adesi F. The risk of new-onset diabetes in antidepressant users-a systematic review and meta-analysis. PLoS One 2017;12(7):e0182088. 
2. Nguyen TTH, Roussin A, Rousseau V, Montastruc JL, Montastruc F. Role of serotonin transporter in antidepressant-induced diabetes mellitus: a pharmacoepidemiologicalpharmacodynamic study in VigiBase ${ }^{\circledR}$. Drug Saf 2018;41(11):1087-96.

3. Gafoor R, Booth HP, Gulliford MC. Antidepressant utilisation and incidence of weight gain during 10 years' follow-up: population based cohort study. BMJ 2018;361:k1951.

4. Gibson-Smith D, Bot M, Milaneschi Y, Twisk JW, Visser M, Brouwer IA, et al. Major depressive disorder, antidepressant use, and subsequent 2-year weight change patterns in the Netherlands Study of Depression and Anxiety. J Clin Psychiatry 2016;77(2):e144-51.

5. Shi Z, Atlantis E, Taylor AW, Gill TK, Price K, Appleton S, et al. SSRI antidepressant use potentiates weight gain in the context of unhealthy lifestyles: results from a 4-year Australian follow-up study. BMJ Open 2017;7(8):e016224.

6. Azevedo Da Silva M, Balkau B, Roussel R, Tichet J, Fumeron F, Fagherazzi G, et al. Longitudinal association of antidepressant medication use with metabolic syndrome: results of a 9-year follow-up of the D.E.S.I.R. cohort study. Psychoneuroendocrinology 2016;74:34-45. Epub 2016 Aug 21.
7. Fjukstad KK, Engum A, Lydersen S, Dieset I, Steen NE, Andreassen OA, et al. Metabolic abnormalities related to treatment with selective serotonin reuptake inhibitors in patients with schizophrenia or bipolar disorder. J Clin Psychopharmacol 2016;36(6):615-20.

8. Breeden M, Brieler J, Salas J, Scherrer JF. Antidepressants and incident hypertension in primary care patients. J Am Board Fam Med 2018;31(1):22-8.

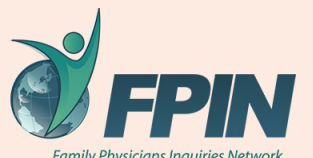

Family Physicians Inquiries Network

Clinical Inquiries are author-formulated questions that are answered with the best available current evidence, written by family medicine residency faculty and their residents through the Family Physician Inquiries Network. The strength of recommendations and the level of evidence for individual studies are rated using criteria developed by the EvidenceBased Medicine Working Group (www.cebm.net). The Family Physicians Inquiries Network Clinical Inquiries series in Canadian Family Physician is coordinated by Rick Guthmann, MD, MPH, Editor-in-Chief for the series. If interested in submitting questions or writing answers for this series, go to www.fpin.org or e-mail ci@fpin.org. () Family Physicians Inquiries Network. Used with permission. 